\title{
Significado del proceso de diseño en la formación del arquitecto. Particularidades en Cuba.
}

\author{
The meaning of the design process in the training of the architect. Particularities \\ in Cuba.
}

Recibido: Junio 2018

Aceptado: Agosto 2019

\section{Resumen}

El proceso de diseño en arquitectura es sin duda una compleja operación intelectual, espiritual, imaginaria y virtual, cuyos resultados se expresan mediante la modelación, consistente en dibujos, maquetas, memorias descriptivas etc., que permite hacer realidad una obra de edificación. Siempre se está anticipando e imaginando un resultado, aún incluso antes de iniciar el proceso diseñador propiamente dicho. Entender el proceso de diseño en arquitectura, ayudará a imaginar y a poner en marcha nuevos procesos pedagógico-docentes orientados hacia el aprendizaje de aquellos conocimientos, habilidades, competencias y desempeños conducentes a un manejo más pleno del proceso diseñador.

El texto tiene la intención de destacar el proceso formativo del arquitecto centrado en el conocimiento de métodos que se utilizan para estructurar la secuencia lógica del proceso de diseño, como elementos centrales para su futuro desempeño profesional en el perfil de proyectista. Se destaca cómo la formación permite que se adquieran competencias que conllevan a imaginar, idear y diseñar los espacios que conforman los proyectos de arquitectura, para luego materializarlo en obras arquitectónicas. En tal sentido el proceso de diseño es también una forma de conocimiento, de imaginación creadora, de reflexión y de anticipación.

\section{Palabras Clave:}

Formación del arquitecto; Proceso de diseño; Método de diseño.
Aymeé Alonso Gatell ${ }^{1}$

\begin{abstract}
The process of design in architecture is a complex intellectual, spiritual, imaginary and virtual operation without a doubt, the results are express by modelation, consisting of drawings, maquettes, descriptive memoirs etc, That allows doing reality a work of edification. Anticipation and imagination are always present, even before the design process began. Understanding the process of design in architecture, will help to create and to get starting new pedagogic teaching processes guided to the learning of knowledge, abilities, competitions and conducive performances to a most complete handling of the designer process This text has the intention to highlight the formative process of the architect focusing in the knowledge of methods that are use to structure the logical sequence of the design process, has central elements for his future professional performance in planner's profile. . Stands out how the formation allows to acquire competitions that bear to imagine, to dream up and to design the spaces that conform the architectural projects, for later materialize it in architectonic works. The design process is also a form of knowledge, of imagination, reflection and anticipation.
\end{abstract}

\section{Keywords:}

Formation of the architect; Design process; Methods of design.

\footnotetext{
${ }^{1}$ Nacionalidad: cubana; adscripción: Universidad de Camagüey; aymee.alonso@reduc.edu.cu
} 


\section{Antecedentes y precisiones conceptuales.}

En la actualidad se han dado importantes pasos para comprender y explicar cómo opera el proceso de diseño en sí. El concepto de proceso de diseño integrado (Trebilcock, 2009), aparece definido como algo esencial para alcanzar estándares de sustentabilidad y eficiencia energética en la arquitectura. Este autor plantea que el proceso debe evolucionar de un modelo tradicional donde el proyecto transita de forma lineal, que parte desde el arquitecto y seguidamente a los especialistas, hacia un proceso integrado donde todos los miembros del equipo de diseño trabajan en forma colaborativa desde los inicios.

Por otra parte, Timmling (2009) sintetiza en su trabajo "Reflexiones en torno al proceso de diseño en arquitectura" las opiniones de cinco grandes "maestros" de la arquitectura internacional: Frank Lloyd Wright, Le Corbusier, Walter Gropius, Frank Gehry y Oscar Niemeyer como reflexiones metafóricas que evidencian las complejidades que acompañan al proceso de diseño en arquitectura. En todas ellas se hace mención a algunos de los principios primordiales que las rigen y estructuran, como son la necesidad de tener "visiones de futuro", de considerar lo "impredecible" y el transformar la "incertidumbre" en una oportunidad diseñadora.

Otros principios implícitos son el de la "incógnita recurrente", la importancia de adoptar una "conciencia auto-evaluativa y crítica", la necesidad de trabajar con "certezas verificables y relevantes" y el contar con referentes valóricos que sean social y culturalmente representativos y transversales. Siempre confiar en la creatividad y nunca dejar de proponer innovaciones, que las sociedades tanto necesitan para tener éxito en sus desarrollos.

\section{El proceso de diseño en la enseñanza de la arquitectura.}

Parte del proceso de formación de los estudiantes de arquitectura para un futuro ejercicio profesional incluye considerar que, si bien no construye o determina la forma de la ciudad, puede con sus diseños crear los espacios urbanos y/o transformarlos, generando un impacto ambiental que puede llegar a ser positivo o negativo. De allí la necesidad de abordar los problemas de la ciudad a partir de la comprensión de conceptos y herramientas del diseño urbano (Trebilcock, 2009). En tal sentido, el proceso de aprendizaje del diseño urbano-arquitectónico, resulta complejo tanto en su aspecto operativo y metodológico, como en su aspecto conceptual.

El proceso de diseño permite la significación y la transformación de la realidad. Produce obras concretas, pero igualmente busca que el usuario se emocione con los valores y cualidades formales que estas obras proporcionan, por medio de vivencias espaciales y de uso. Estas experiencias conducen a la creación de imaginarios culturales comunes y al surgimiento de identificaciones de pertenencia y de integración social. El proceso de diseño representa también una forma de actuar, de intervenir y de transformar el mundo. En dicho proceso se concatenan, simultánea o secuencialmente, procedimientos y operaciones con metas y objetivos y, ellos a su vez, con expectativas y visiones de futuro. Esto incluye valores y motivaciones, siempre en la perspectiva de lograr calidad, confiabilidad y concordancia en los resultados. Es parte fundamental de este proceso la visualización de las consecuencias y los impactos en los contextos, sean ellos territorialespaciales, sociales, económicos, culturales o medioambientales, cuyas implicaciones desbordan y muchas veces superan el accionar del propio diseñador.

A la vista de lo descrito, se debe potenciar la comprensión volumétrica de las formas, la percepción espacial de los modelos virtuales y el desarrollo de la creatividad unida al pensamiento crítico (Redondo, E, el al, 2012). El medio digital aparece como un entorno adecuado para propiciar el conocimiento. La impartición de la docencia con ayuda de las TIC acompañada del dibujo tradicional, facilita el interés y las aptitudes del alumnado en el proceso creador (Rodríguez, M.L, y Ricardo, L, 2007). Otros autores (Alonso, A, Leyva, C. L, y Campos, E, 2012), aportan en el contexto de la enseñanza de la arquitectura sobre el empleo de las herramientas digitales para favorecer la formación del estudiante y la adquisición de actitudes creativas y comprometidas con la mejora del entorno.

El proceso de diseño desarrolla capacidades reflexivas, predictivas, creativas e innovativas. En su capacidad integradora, asocia factores y circunstancias diversas y además construye líneas 
o cadenas lógicas de acción, que van buscando y encontrando un cauce de salida a propuestas y procedimientos. Una amenaza se transforma en una oportunidad si nuestras visiones y predicciones son inspiradoras, creativas e innovadoras. El proceso de diseño representa el "aprender aprendiendo" y de esa manera desencadena un "saber haciendo cada vez más predictivo y estratégico" declarándose como una forma de aprendizaje extraordinariamente efectiva para modelar, configurar y transformar el mundo.

Pérez (2004), define el método de diseño no como resultado aislado o final, sino como un proceso, o sea una secuencia íntegra de pasos en etapas y fases dirigidos a la solución del problema (las necesidades). La realidad que enfrenta el diseñador es compleja y variada, de hecho, el objeto real de la profesión es dinámico, y para su conocimiento y acción proyectual es válido el empleo de diferentes métodos específicos, aunque en el diseño arquitectónico se trata de unificar una concepción general según el "Método General para el análisis de la realidad y el proyecto", como plantea el programa de la Disciplina de Diseño Arquitectónico y Urbano y el plan de estudios en Cuba.

Los métodos de diseño expresan posiciones ideológicas, filosóficas y epistemológicas de sus autores. La base del método de diseño arquitectónico que se aplica en la enseñanza en el país, está en correspondencia con la concepción científica del mundo, con el método científico y en particular, dado el carácter altamente socializado del objeto de la profesión, se relaciona con el método de investigación de las ciencias sociales.

Otros autores (Briceño Ávila, Morella, Gómez Rosales, Luz J, 2011), conciben el proceso de diseño desde la perspectiva de favorecer una discusión permanente entre especialistas, cuya área de interés específica es precisamente aquella referida al estudio del hecho arquitectónico, no como un elemento disociado de su entorno, sino como un componente más de un conjunto mayor. Este enfoque desde el punto de vista conceptual, tiene su pretexto en que las secuencias espaciales, el respeto por la diversidad, y un férreo sentido de la identidad cultural, se erigen como condiciones siempre y cuando den una respuesta proyectual urbana. La idea fundamental de los autores sobre la cual se sustenta el proceso de diseño, es entender a la arquitectura como conformadora del lugar en el que se desarrolla la actividad del hombre. Este lugar no es más que la ciudad, definida, física y espacialmente, por los objetos arquitectónicos y sus interrelaciones.

Los antecedentes de los métodos de diseño y los de enseñanza actual se ubican en las primeras décadas del siglo XX, relacionados con los cambios que ocurrían en la arquitectura y el diseño en esa época. La experiencia de la Bauhaus en 1919 fue un punto clave, pues en el propósito de integrar arte y técnica desarrollaron nuevos métodos para el diseño. Las posiciones academicistas que prevalecían hasta el momento fueron sustituidas por el gradual ascenso del denominado Movimiento Moderno; se niegan los elementos de estilo y los métodos academicistas conservadores y se plantea un método basado en la integración de aspectos como los funcionales, constructivos y estéticos. Se concibe que el resultado del diseño sea determinado por factores condicionantes, y a partir de la función del edificio.

Walter Gropius fue uno de los principales arquitectos y docentes que aportó a los métodos de diseño, cuando emigra a EUA por el ascenso del fascismo en Europa y la II Guerra Mundial, plantea aún estas ideas: “...introducir un método de acercamiento que permita hacer frente a los problemas según sus peculiares condiciones...", a los jóvenes arquitectos, crear "...de acuerdo con las condiciones técnicas, económicas, y sociales en las que se encuentra..."

En los años '60, en la carrera de arquitectura en La Habana se aplicó en el método de diseño un esquema propuesto por el profesor de origen español Joaquín Rallo. Este se centraba fundamentalmente en la parte del análisis de los factores del diseño. Los factores a analizar para un problema de diseño se integraban por:

- Análisis conceptual

- Análisis funcional

- Análisis ecológico

- Análisis técnico-constructivo

- Análisis formal

Estos aspectos tienen vigencia y utilidad, y aún se mantienen como una base para desarrollar el análisis de los proyectos arquitectónicos en la carrera de arquitectura.

Posteriormente se dieron influencias de las corrientes cientificistas que se daban a nivel internacional sobre los métodos de diseño. En tal sentido en Cuba se publicaron textos de autores como Broadvent y Best, en un material docente compilado por la Universidad Central de Las 
Villas en la década de los años '70 bajo el título Metodología del diseño arquitectónico.

También se aplicaron concepciones del método de Rozas (1976), quien publicó un material sobre el tema; este incluía aplicación de elementos matemáticos en el diseño como los grafos y matrices, las cuales se usan actualmente en el diseño para el análisis funcional y la organización espacial de un programa arquitectónico dado, aunque se mantiene también el esquema de factores de Rallo. Por otra parte, no todos los factores de diseño se pueden parametrizar matemáticamente.

Los contenidos sobre método de diseño fueron tratados desde la década del ' 60 en tres vertientes principales:

- A través de las asignaturas de Diseño Básico previas a las asignaturas de proyecto: manejaban los aspectos formales y espaciales del diseño.

- Asignaturas de Historia de la Arquitectura: manejaban los aspectos de significados culturales y condicionamiento social, además de otros factores condicionantes (circunstancias generales y específicas).

- Asignaturas de Proyecto: manejaban los aspectos funcionales del diseño.

En los años '90 con la aplicación de un nuevo Plan de Estudios, se comienza un proceso de mayor integración interdisciplinaria de la carrera, y a partir de este momento, con sucesivos perfeccionamientos ha resultado un factor clave para el proceso docente educativo de la carrera, hasta la actualidad. Una característica de este perfeccionamiento curricular es la unificación en una concepción metodológica integral para las asignaturas de diseño de la carrera en sus diferentes años. Por supuesto que existen particularidades según cada semestre en cuanto a nivel de desarrollo de las habilidades, tipo de obra a proyectar, asignaturas que se integran etc.

En este sentido, se consideran adecuadas las perspectivas de Granados, M (2017) y Miranda, $\mathrm{V}$ y Esteves, M (2018), quienes enfatizan en sus respectivos estudios, que para que la interdisciplinariedad surta efecto debe haber un cambio profundo que sea definitivo en la manera de pensar las disciplinas en sí y la producción de conocimiento que de ellas se deriva, en el caso del último texto, lo contextualizan desde la propia arquitectura.

En la década mencionada se convocaron en el Instituto Superior Politécnico "José Antonio Echeverría (ISPJAE) de La Habana dos eventos sobre enseñanza de la arquitectura; en el de 1995, el II Seminario Internacional Pedagogía de la Arquitectura, se apreciaron dos enfoques metodológicos sobre el diseño:

a-El enfoque científico-metodológico: caracterizado por el uso de métodos de diseño, e integración de otras disciplinas.

b- El enfoque intuitivo-tipológico: caracterizado por aspectos formales, por procesos intuitivos y por el papel importante dado a la tipología, los arquetipos, los modelos. (ver Gráfico 1).

Gráfico 1. Ejemplo de método basado en el enfoque intuitivo-tipológico.

Fuente: Basado en Conferencia de proyecto arquitectónico, $\mathrm{Dr}$. C. Oscar Prieto Herrera, Universidad de Camagüey, 2005.

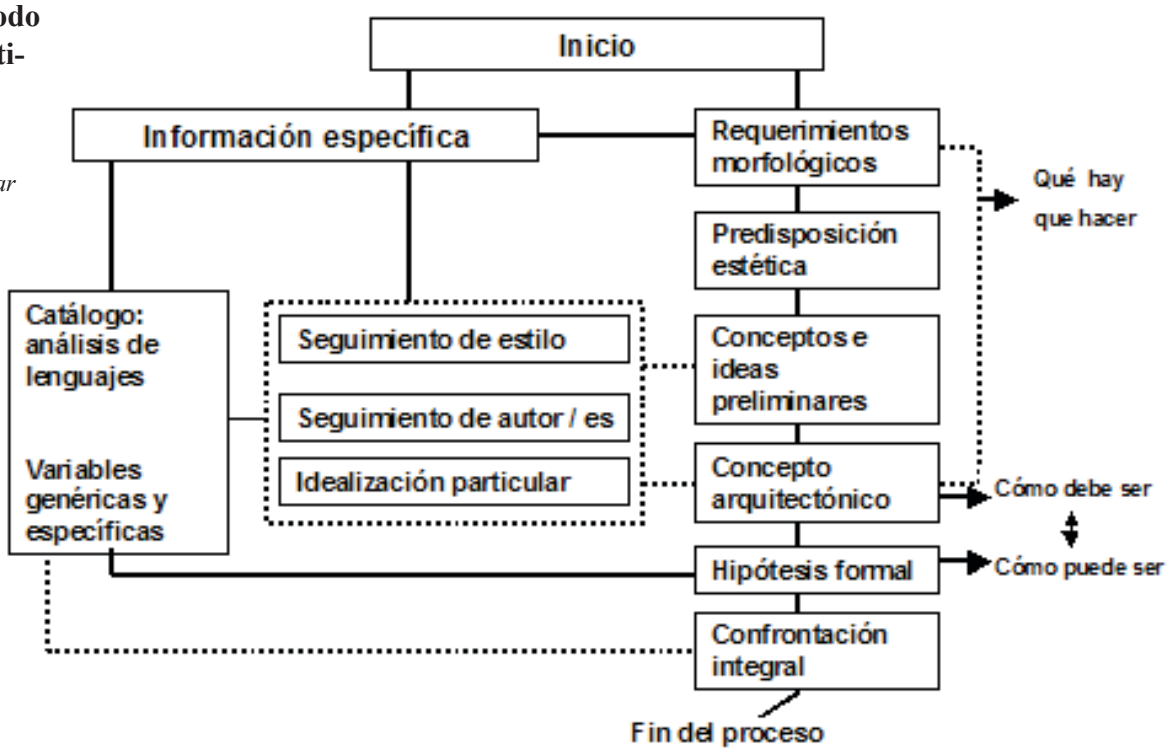


Aunque en las definiciones conceptuales se ha referido el término método de diseño, consecuente con el plan de estudios vigente en Cuba, los autores de esta investigación coinciden con Pérez (2004), al concluir que en realidad lo que se aplica en la disciplina de Diseño Arquitectónico y Urbano es una metodología, considerando este término en su acepción como un conjunto de métodos, tanto teóricos como empíricos. En cierta medida esta metodología se puede calificar como ecléctica, posee un basamento científico, pero armoniza métodos teóricos, racionales, con métodos del conocimiento empírico, intuitivo. La aplicación de esta metodología ha permitido dar un salto cualitativo en la formación de los futuros profesionales de la carrera de arquitectura.

\section{Análisis sobre métodos de diseño.}

La arquitectura se rige por principios básicos, estos principios son:

1. El principio de sistemas

2. El principio de subordinación a factores condicionantes

3. El principio del proceso de diseño

4. El principio de la significación

5. El principio de la estructura en imágenes (que explica una relación entre pensamiento lógico-científico y el pensamiento estructurado en imágenes).

Lo relacionado con método de diseño se incluye con mayor significación en el principio No.3, o sea el método es la herramienta teóricoconceptual y práctica para desarrollar el proceso de diseño. Aunque lo metodológico sobre diseño se relaciona con los demás principios.

En el contexto de la diversidad de autores, existen métodos de diseño arquitectónico enfocados hacia ciencias sociales, algunos tratan de aplicar las ciencias exactas como la matemática y la cibernética. Otros consideran aspectos más subjetivos o intuitivos de la creación; no son métodos basados en la lógica del pensamiento científico, sino en el pensamiento intuitivo, en el conocimiento artístico, la inspiración y la creatividad propia de las artes. Esto es reflejo de las polémicas sobre la arquitectura concebida como arte o como técnica. De hecho, la arquitectura resulta doblemente relacionada con el conocimiento artístico y con el conocimiento científico, aunque el siglo XX fue el momento de cambios que enfocaron la arquitectura como ciencia, y esto se reflejó también en los métodos de diseño y en la enseñanza de la carrera.

En el desarrollo de los métodos de diseño se han enfrentado las dos tendencias contradictorias antes mencionadas: las que enfatizan la intuición y las que enfatizan la cientificidad; esto no significa que la segunda tendencia sea siempre acertada. El fetichismo científico-técnico ha absolutizado lo técnico y ha hecho separación de los componentes humanistas de los científicostecnológicos, fenómeno que ocurrió en países capitalistas industrializados en los años 60 y 70 del siglo XX.

La clasificación general de las principales tendencias de los métodos de diseño se basa en la manera en que actúa el diseñador, tradicionalmente se clasifican las siguientes, que fueron resumidas en la mencionada publicación docente "Metodología del diseño arquitectónico":

Diseñador como "caja negra" : diseñador como sistema cerrado, se produce un salto creativo, predomina el pensamiento intuitivo

Diseñador como "caja de cristal": sistema transparente, se exterioriza el proceso (es observable y explicable), sigue pasos ordenados: información, análisis, síntesis, evaluación

Diseñador como sistema auto-organizado: un proceso auto dirigido hacia la meta final, es una búsqueda de la solución adecuada y control del sistema de búsqueda, se potencia el proceso de toma de decisiones, existe total control del proceso

El método de diseño aplicado en la docencia tiene como una de sus características principales la estructuración como un proceso que tiene una serie de pasos lógicos:

Información $>$ Análisis $>$ Síntesis $>$ Evaluación $>$ Retroalimentación

Estos pasos pueden aplicarse cíclicamente por niveles de desarrollo de la elaboración del diseño, es decir la retroalimentación da paso a un nuevo ciclo de información, análisis, síntesis, evaluación, pero en niveles más altos de detalle y acabado de la solución de diseño.

Este método analítico-sintético en esencia es de tipo racional. El diseño como actividad creadora en general, tiene características comunes, aunque 
se trabaje en diferentes escalas como son diseño gráfico, industrial, arquitectónico y urbano etc.

A continuación, se presenta la valoración de diferentes métodos como parte de la profundización en el tema, y se presentará también el que se aplica en la Carrera de Arquitectura de la Universidad de Camagüey.

Propuesta metodológica de Fundora.

Fundora $(1986,1988)$ propone lo que denomina "modelo para el proceso creador de la proyección arquitectónica". Este se basa en el método problémico y tiene la siguiente estructura (Ver Gráfico 2).

En este método se aplica la concepción racional, de tipo analítico-sintético, pero además se observa que trata de armonizar el pensamiento lógico-racional y las formas del pensamiento intuitivo, del salto creativo que se produce en el diseñador. Aunque no tuvo la suficiente implementación por parte de los docentes, el método aplicado actualmente tiene rasgos de estos aportes, por ejemplo en aspectos como el squeeze que tienen aplicación para elaborar síntesis parciales del proceso.

Gráfico 2. Modelo para el proceso creador de la proyección arquitectónica.

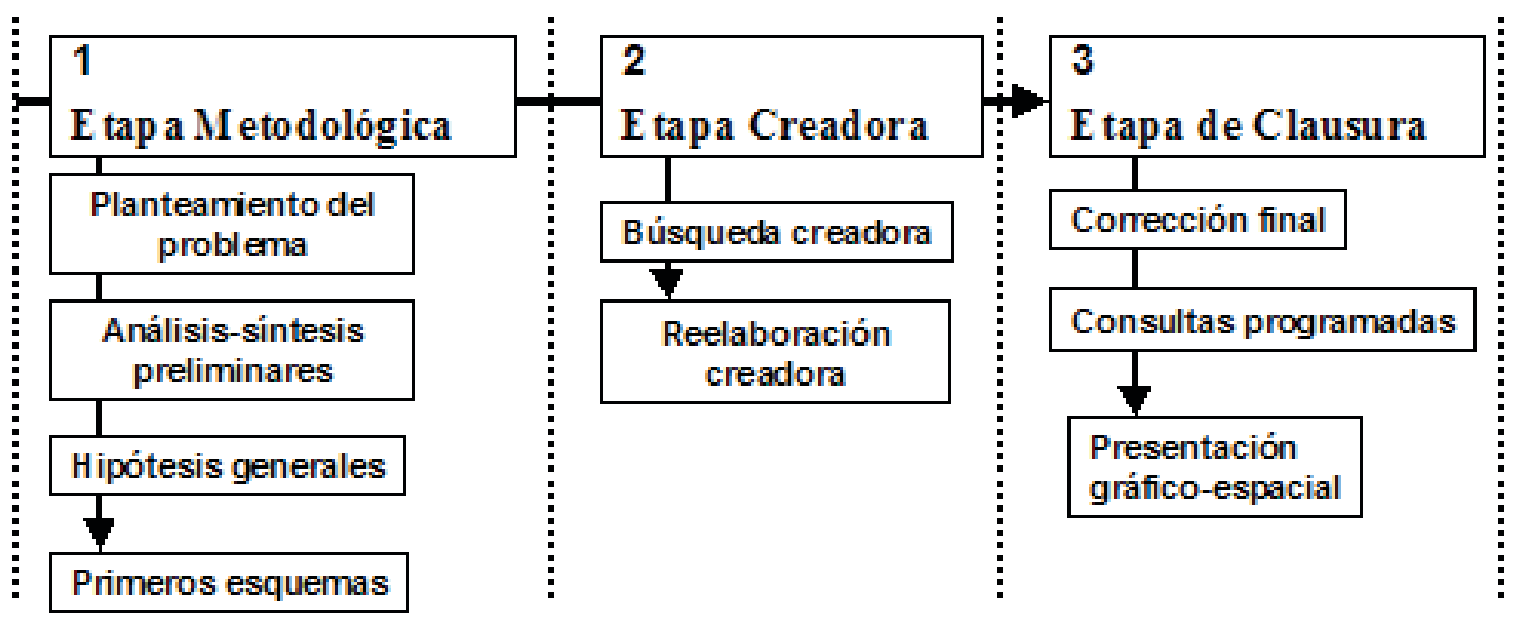

Fuente: Elaboración en base a Fundora (1986, 1988). 


\section{Método de Rozas.}

Similar en concepción al método racional y analítico-sintético propuesto por Fundora, Rozas (1976) elaboró un esquema metodológico para racionalizar el diseño. Como se explicó en el punto de evolución histórica, este incluye aspectos matemáticos tratando de dar exactitud y objetividad.

El modelo de Rozas plantea las cuatro etapas básicas del método analítico-sintético y las desarrolla en cuatro niveles de elaboración del proyecto. A continuación, se muestra el esquema metódico (Gráfico 3).

En el nivel IV se plantean conocimientos más específicos o soluciones detalladas como son los aspectos constructivos, tecnológicos, ecológicos, estéticos, etc.

Gráfico 3. Método analítico-sintético.

NIVEL I DAT OS SOBRE AMBIENTE
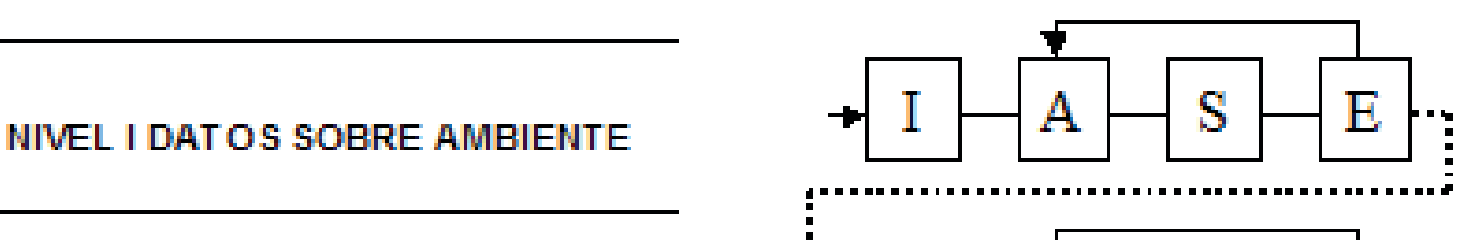

NIVEL II LOCALES-TERRENO-RELACIONES

NIVEL III MATRIZ O RED DE RELACIONES

NIVEL IV ESQUEMA VOLUMÉTRICO

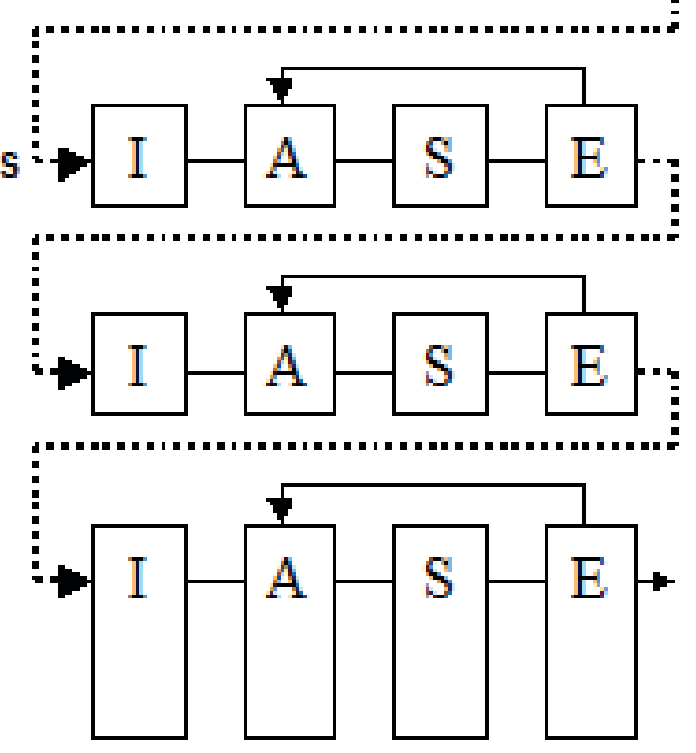

Leyenda: I: Información / A.: Análisis / S: Sintesis / E: Evaluación

Fuente: Rozas (1976) 


\section{Método de Hepler y Wallach.}

Como parte del estudio de fuentes bibliográficas sobre el tema, se analizó la propuesta de estos autores norteamericanos. El texto es de carácter práctico y referido especialmente al diseño de viviendas (Hepler y Wallach 1991), pero es posible extraer la secuencia metodológica que está contenida en el desarrollo de la obra. El modelo propuesto de manera resumida, se muestra en el Gráfico 4.

Como se observa, este método concibe el proceso de manera muy práctica, se inicia con la entrevista al cliente y termina con la construcción de la obra. Una diferencia con el método aplicado en Cuba es en lo que se denomina conceptualización, ya que en el caso cubano el análisis conceptual se considera un momento inicial de la etapa de análisis y en este caso, se denomina así a los pasos posteriores al análisis, por lo que esta es una conceptualización principalmente gráfica a través de bocetos con ideas de solución del proyecto.

Gráfico 4. Método de Hepler y Wallach.

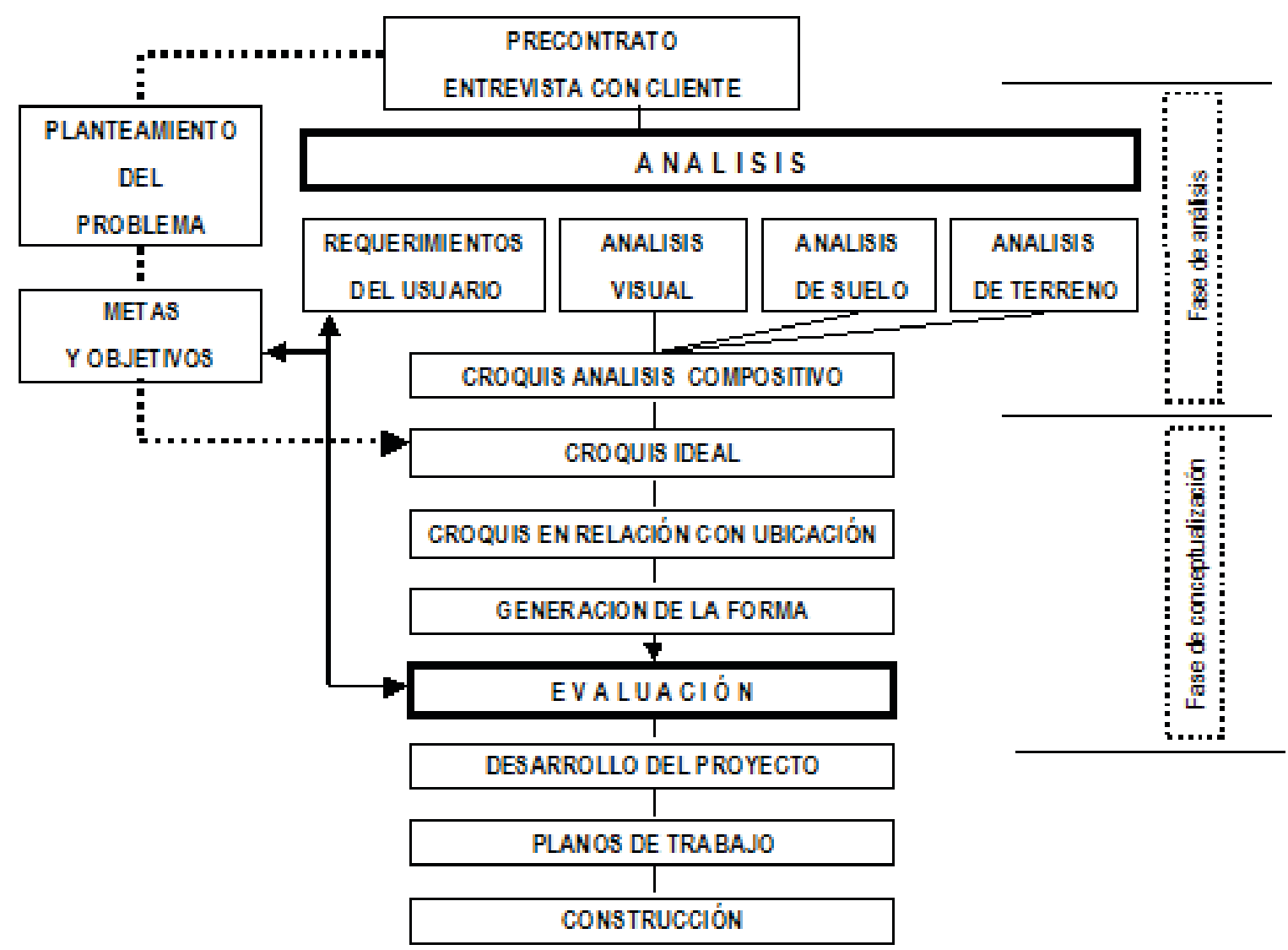

Fuente: Elaboración propia, basado en Hepler y Wallach (1991). 
Método de racionalización de la creatividad: "Creática".

El término de creática trata de definir el método que organiza o sistematiza el pensamiento analítico y el sintético, integrados en el proceso creativo. El esquema presentado por Moya (1991), es un método de creática aplicable a la arquitectura y otras actividades de creación de perfil tecnológico; tiene tres fases principales y cada una se compone de cuatro etapas (Gráfico 5).

En este método de creática, las formas de pensamiento predominantes de las etapas 1 y 3 serían de carácter analítico, y las etapas 2 y 4 de carácter sintético.
A modo de comentarios finales sobre métodos de diseño, se observa que se proponen muchos métodos de diseño que desarrollan alternativas para la etapa inicial de análisis. En algunos casos se conciben procedimientos de análisis relativamente complejos, pero no siempre resulta claramente explicado cómo lograr el salto o transición del análisis a la síntesis, a la idea de diseño como una expresión integrada, con un grado de concreción.

También se observa que algunas disciplinas científicas que se aplican en el análisis de las variables o factores de un problema de diseño, se han desarrollado mucho más como disciplinas en sí mismas. Pero no siempre los diseñadores logran competencias para aplicarlas o incorporarlas de manera asequible a las soluciones de diseño. Aunque en este sentido las herramientas informáticas actuales hacen más viable la aplicación de estos conocimientos científicos a la actividad del creación de espacios arquitectónicos.

Gráfico 5. Método de racionalización de la creatividad, "Creática".

\begin{tabular}{|c|c|c|c|}
\hline ETAPAS & $\begin{array}{c}\text { 1ra fase } \\
\text { cuantitativa }\end{array}$ & $\begin{array}{c}\text { 2da fase } \\
\text { cualitativa }\end{array}$ & $\begin{array}{l}\text { 3ra fase } \\
\text { creativa }\end{array}$ \\
\hline 1 Exploración & $\begin{array}{l}\text { Antecedentes } \\
\text { Causas del problema }\end{array}$ & $\begin{array}{l}\text { Deficiencias } \\
\text { observadas en } \\
\text { soluciones anteriores }\end{array}$ & \begin{tabular}{|l} 
Busca de \\
posibilidades \\
Nuevos elementos \\
capaces de ayudar a \\
esta búsqueda \\
\end{tabular} \\
\hline 2 Estructuración & $\begin{array}{l}\text { Objeto del problema } \\
\text { Entorno } \\
\text { Factores humanos }\end{array}$ & $\begin{array}{l}\text { Ordenación de } \\
\text { deficiencias que } \\
\text { admiten una mejora } \\
\text { inmediata y otras } \\
\text { requieren estudio } \\
\end{array}$ & $\begin{array}{l}\text { Propuesta de } \\
\text { soluciones a } \\
\text { problemas antes } \\
\text { expuestos }\end{array}$ \\
\hline 3 Reducción & $\begin{array}{l}\text { Ordenación de datos } \\
\text { Búsqueda de nuevos } \\
\text { elementos capaces de } \\
\text { formular problema de } \\
\text { otro modo }\end{array}$ & $\begin{array}{l}\text { Relación entre la } \\
\text { nueva formulación y } \\
\text { las deficiencias } \\
\text { ordenadas } \\
(1 \text { ra- } 4) \leftarrow \rightarrow(2 \text { da- } 2)\end{array}$ & $\begin{array}{l}\text { Elección entre las } \\
\text { soluciones }\end{array}$ \\
\hline 4 La idea & $\begin{array}{l}\text { Nueva formulación } \\
\text { ampliada }\end{array}$ & $\begin{array}{l}\text { Nuevo concepto del } \\
\text { objeto estudiado }\end{array}$ & $\begin{array}{l}\text { Resultado final } \\
\text { Comprobación }\end{array}$ \\
\hline
\end{tabular}

Fuente: Elaboración basada en Moya (1991). 


\section{Análisis de ejemplos: métodologias de diseño en ámbitos específicos.}

Ejemplo 1. Método de proyecto en una empresa de Camagüey.

Para la profundización en el tema se incluyó el análisis del modo en que se realizan proyectos en el ámbito profesional. Para ello se seleccionó la Empresa de Proyectos No.11 de Camagüey, Cuba, donde se realiza la actividad de diseño más compleja en proyectos de obras turísticas, hospitales, escuelas y otros programas.

Por medio de entrevista a especialistas de la entidad se determinó cómo se organiza este proceso, en particular el diseño de obras de turismo, fundamentalmente hoteles y villas turísticas de playa y de ciudad (ver Gráfico 6).
En este método está presente el esquema básico conocido, de información - análisis - síntesis - valoración. Los diferentes pasos se corresponden con el momento o nivel de complejidad por el que transita la elaboración del proyecto. El análisis conceptual incluye los diferentes factores de diseño. En la valoración participan diversas entidades como el proyectista general y su equipo, el inversionista, y un grupo que representa al Ministerio del Turismo en Cuba. En relación con la información que aporta el inversionista, se observa que no sólo plantea requerimientos funcionales del programa, sino que establece algunos criterios de estilo. Esto implica que el resultado de la expresión formal tiene influencia y en alguna medida es predeterminado por el inversionista, y el diseñador debe lograr las formas que responden a los criterios que le han planteado.

Gráfico 6. Proceso de diseño para obras del turismo. Empresa de Proyectos No. 11 de Camagüey.

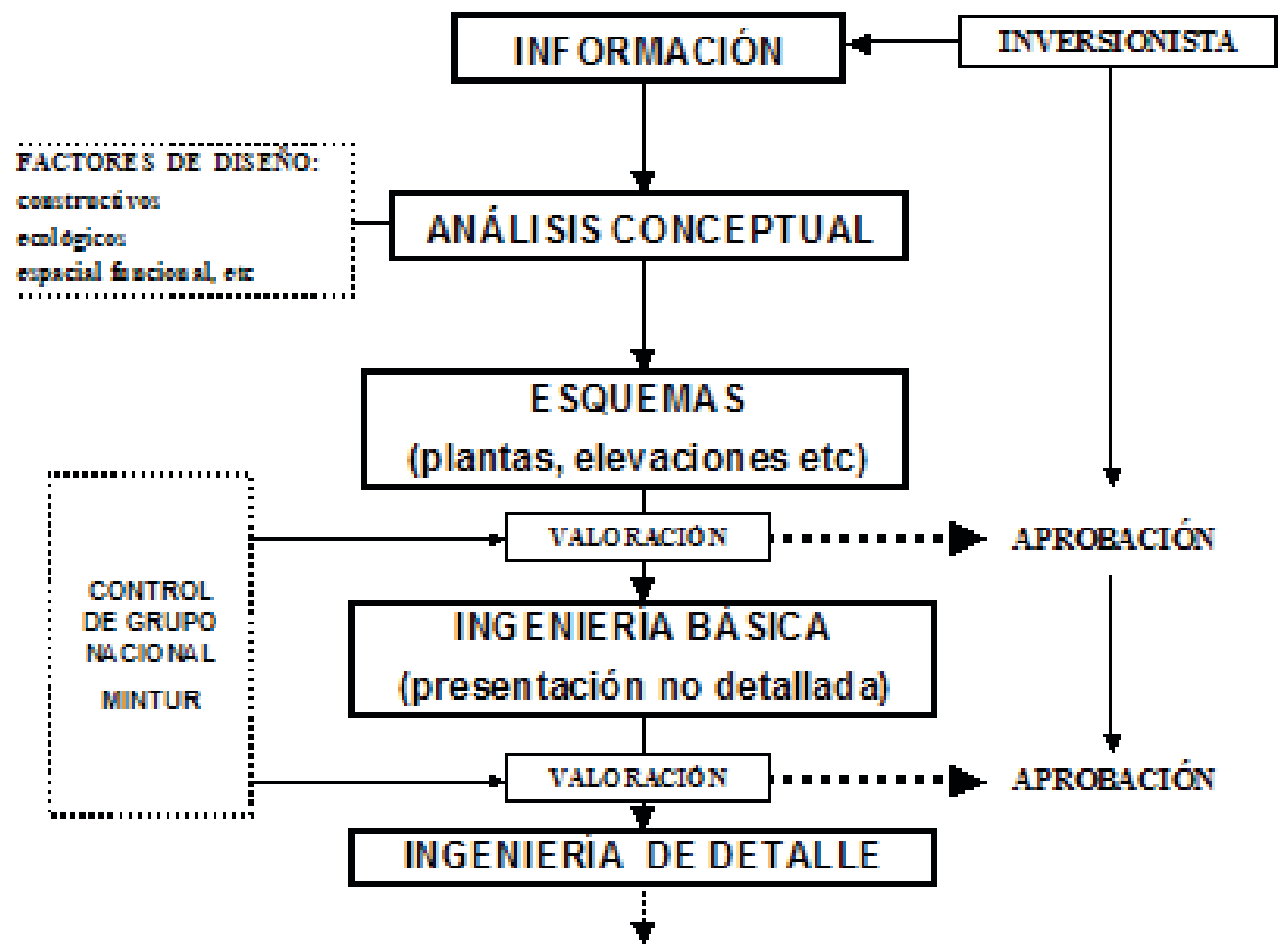

Fuente: Elaboración propia, en base a la información de proyectistas de la empresa. 
Ejemplo 2. Método de diseño para proyecto de viviendas, en la docencia de arquitectura (ISPJAE La Habana).

El ejemplo que se presenta es la organización del proceso de diseño para un proyecto de viviendas en una zona existente. Este fue elaborado por estudiantes de Arquitectura del ISPJAE, dirigido por la Dra. Dania González Couret. Estudios posteriores (González, D., 2012 y 2015), valoran la evolución del pensamiento académico sobre el tema de la vivienda, materializado en los enfoques teóricos y metodológicos desarrollados en la Facultad de Arquitectura de La Habana (Gráfico 7).

Gráfico 7. Método de diseño para proyecto de viviendas, equipo de estudiantes ISPJAE La Habana.
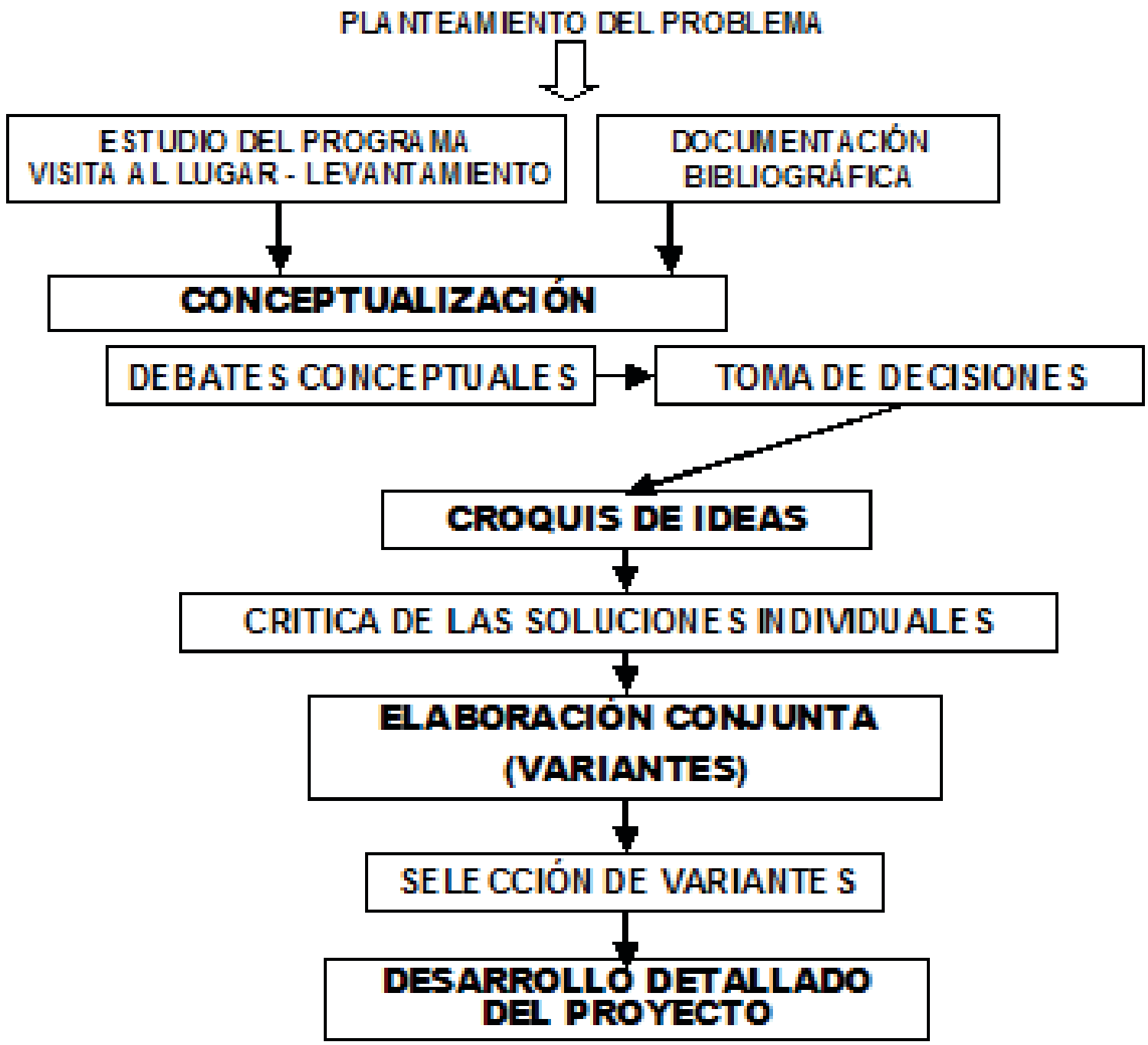

Fuente: Elaboración propia, en base a la información de los proyectos de estudiantes. 
Ejemplo 3. Método aplicado en la docencia en Universidad Autónoma de Puebla, México.

El ejemplo que se presenta corresponde a un proyecto de octavo semestre de la carrera de arquitectura de la BUAP. El proyecto consiste es parte de la tesis como ejercicio de titulación de licenciatura en arquitectura.

En este caso, el método planteado busca la solución de manera más directa o intuitiva, y se exterioriza menos en los pasos detallados que se siguen en el proceso. El tipo de proyecto es de alojamiento turístico en zonas de playa (Gráfico 8).

En este método, se observa que hay elementos de la tendencia o enfoque intuitivo-tipológico, caracterizado por aspectos formales, por el papel importante dado a la tipología (los arquetipos, los modelos). Por ejemplo, en el paso de criterios de composición, los alumnos seleccionan una tipología arquitectónica determinada y trabajan con esos códigos. Por otra parte, para la implantación del conjunto en el terreno asignado hacen una geometrización de la parcela buscando ejes, trazados interiores, particiones y otros elementos geométricos que le sirvan de base para el plan general de la obra. Este procedimiento puede facilitar el trabajo compositivo a escala de la parcela, pero también resulta algo rígido en algunos casos, al forzar la solución espacial arquitectónica a una geometría predefinida.

En el aspecto didáctico desarrollan el proyecto bajo asesoría de tres docentes diferentes, cada uno representando tres ejes: tecnología, diseño y teoría. Estos docentes desempeñan también en el método de diseño la función de valoración de etapas parciales.

Gráfico 8. Método aplicado en un proyecto de obra de turismo en Universidad Autónoma de Puebla, México.

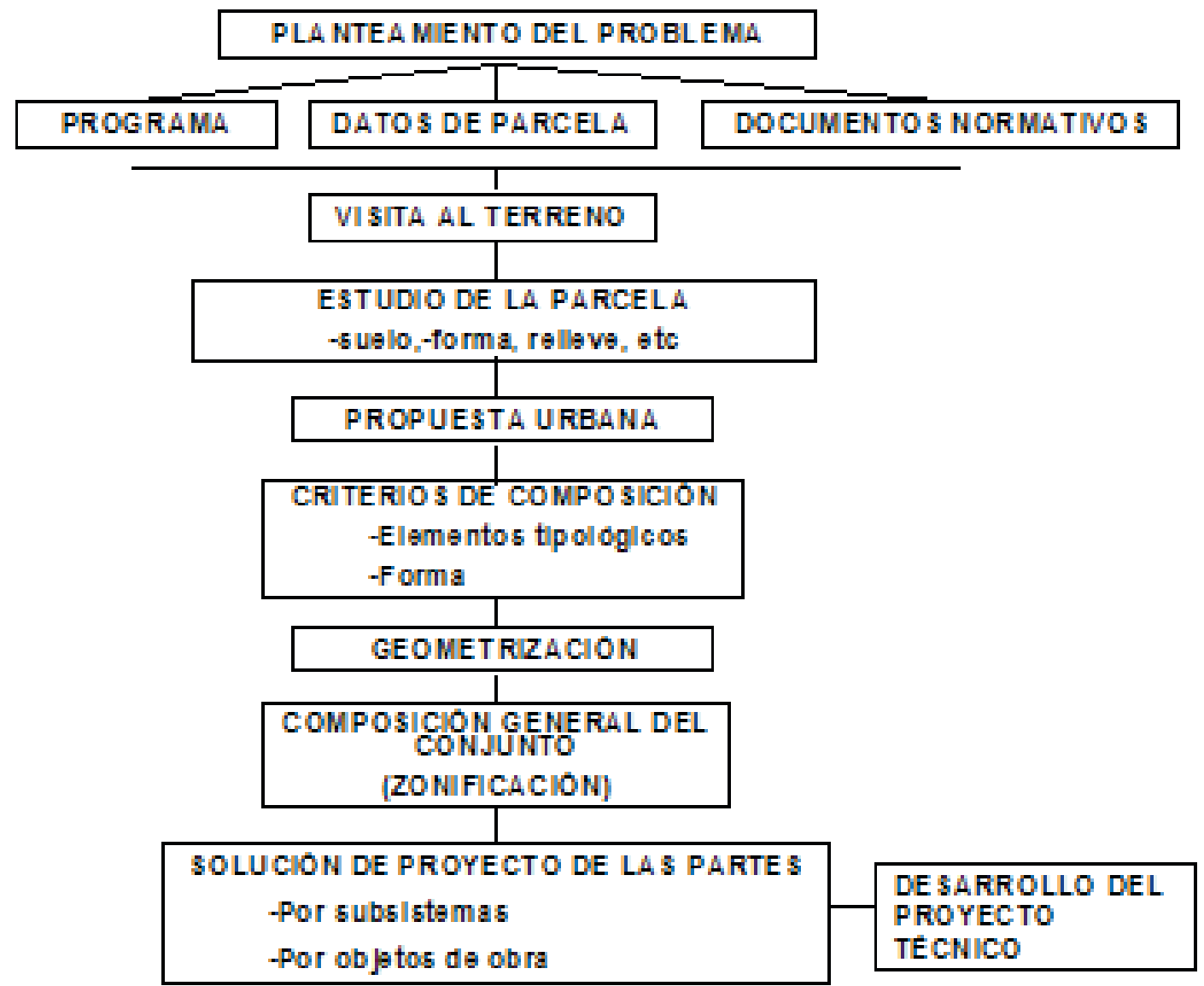

Fuente: Elaboración en base al análisis de proyectos de estudiantes de arquitectura de octavo semestre, entre 2004-2006. 
Ejemplo 4. Método de diseño en la Disciplina de Proyecto Arquitectónico y Urbano. Universidad de Camagüey. Cuba.

El método que se aplica en la carrera de arquitectura, a partir de los proyectos de segundo año, de manera general se corresponde con el siguiente esquema (Gráfico 9).

En este método están presentes en general los principios del método analítico-sintético antes expuesto, aunque para cada año, según el problema profesional a resolver y el nivel del año tendrá sus adecuaciones de implementación.
Por otra parte, en las condiciones académicas de formación en la universidad, los aspectos detallados del método tienen un tratamiento más minucioso con el fin de su aprendizaje. Es así que la Fase 1 de conceptualización, se trabaja de manera detallada (Gráfico 10).

Gráfico 9. Método de diseño, Disciplina de Proyecto Arquitectónico y Urbano. Universidad de Camagüey. Cuba.

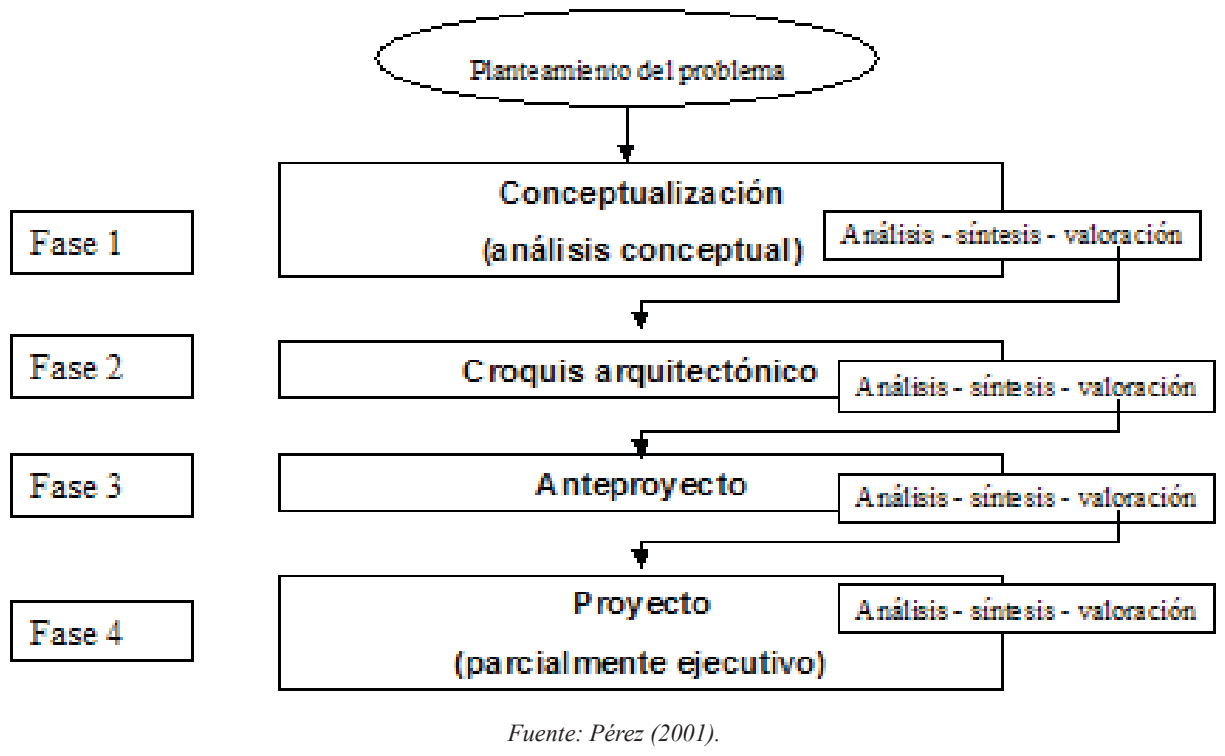

Gráfico 10. Fase de conceptualización.

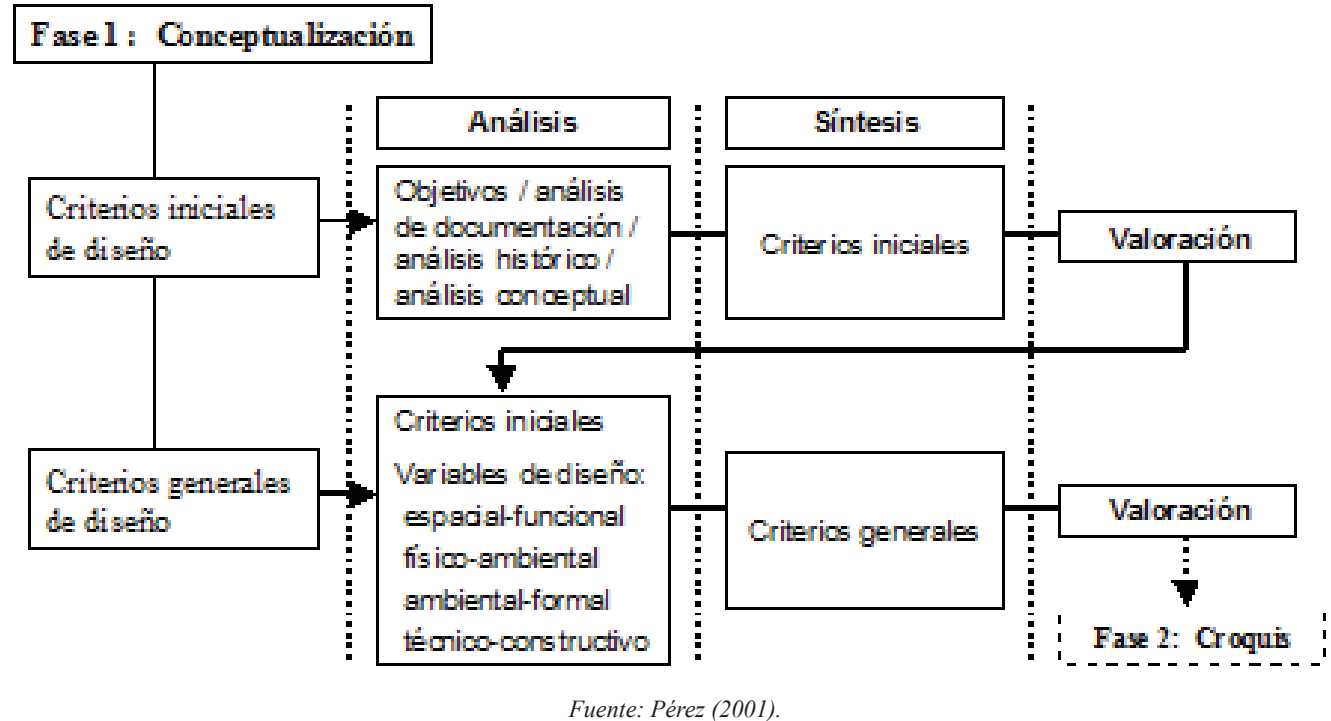


Esta fase se elabora por medios verbales y gráficos, y se supone que tiene gran importancia para el desarrollo ulterior del proyecto. En algunos casos la deficiencia está en que los estudiantes analizan factores, pero no logran sintetizar en la fase de conceptualización, o sea no logran cristalizar verdaderos criterios de diseño. En ejemplos estudiados, se observa una tendencia a conceptualizar mediante el lenguaje gráfico, lo que es positivo en el estudiante pues lo acerca más al objeto de la profesión. No se debe confundir conceptualización con explicaciones verbales puras, ya que esta también puede expresarse estructurada en imágenes, combinada con las formas verbales.

En relación con este último ejemplo, más cercano a la experiencia docente de los autores, se comenta sobre aspectos cognoscitivos y de las habilidades que se forman cuando se sigue el método de diseño.

En principio los métodos de diseño arquitectónico también están relacionados con los procesos cognoscitivos, que en el sujeto se manifiestan en su relación con la realidad externa, y desde allí al reflejo interno para construir un conocimiento, tomar decisiones, imaginar la solución del problema. Y nuevamente se regresa a lo externo cuando su actividad de diseño se expresa en el lenguaje del diseñador, a través de dibujos, maquetas, croquis, memorias, calculos etc. el estudiante expresa el resultado.

Pero en el diseño podemos plantear que existe una contradicción muy importante cuya implicación se palpa en los resultados concretos de los alumnos. Dado que el método de diseño es analítico-sintético en esencia, la tendencia regular del estudiante es desarrollar la etapa analítica por procedimientos verbales, por pensamiento lógicoracional procesando información en forma de documentos escritos, organigramas, datos técnicos, esquemas simbólicos y otras formas. Pero en la síntesis, dado que el objeto de la profesión tiene una estructura en imágenes, una manifestación espacial, es sumamente difícil para algunos producir el salto de lo verbal a lo gráfico espacial.

Las situaciones de insuficiencia del aprendizaje observadas confirman esta dificultad, así por ejemplo se dan algunas como las siguientes:

- Algunos estudiantes descomponen los factores que determinan en su diseño y desarrollan el análisis de los mismos relativamente bien, pero al integrarlos y traducir esto en la síntesis gráfico-espacial, esta no se corresponde con el análisis y tiene pobre calidad. Y paradójicamente puede no tener relación, por ejemplo cuando el estudiante analiza requerimientos del factor de acondicionamiento ambiental que debe cumplir su proyecto, y al representarlo en la síntesis gráfica del edificio no se cumplen los requisitos declarados. Esto por supuesto no es un problema puramente cognoscitivo del estudiante, es también un problema didáctico a resolver por el profesor con los métodos de enseñanza aprendizaje.

- Otros estudiantes son reacios a pensar aplicando habilidades de razonamiento lógico en la etapa analítica, como la comparación, clasificación, relación entre variables, jerarquizar decisiones a tomar, etc., y en lugar del razonamiento previo para obtener una decisión de diseño, tienden a un automatismo gráfico y plantear algún bocetaje intuitivo aproximado desde un punto cero, para perfeccionar la solución, generalmente deficiente, mediante un método empírico similar al de ensayoerror o el de diseñador como caja negra que espera un salto imaginativo. Pero el proyecto de arquitectura no es una actividad propiamente de inspiración creativa artística, ya que opera con variables que se expresan en datos, magnitudes, requisitos de funcionamiento del edificio que exigen un análisis para buscar las soluciones más adecuadas.

\section{Conclusiones.}

El tema analizado permitió evidenciar la importancia y la complejidad que tiene en la Disciplina de Proyecto Arquitectónico y Urbano lo relacionado con el método de diseño. El llamado Método general de análisis de la realidad y el proyecto, aún requiere mayor grado de elaboración para integrarlo como el esquema conceptual-metodológico rector del diseño.

Los contenidos sobre método de diseño son menos trabajados que los contenidos que reflejan temas específicos del objeto de la profesión; en este sentido la metodología de diseño debe ser asimilada en su esencia, en el modo de actuación del profesional.

Aunque en las definiciones conceptuales se ha referido durante todo el trabajo el término método de diseño, consecuente con lo planteado, se puede concluir que en realidad lo que se aplica en la disciplina de Proyecto Arquitectónico y Urbano 
es una metodología, considerando este término en su acepción como un conjunto de métodos.

La metodología aplicada en Cuba puede calificarse como ecléctica, posee un basamento científico, pero armoniza métodos teóricos, racionales, con métodos del conocimiento empírico, intuitivo. Por ejemplo: el renovado interés que se le da nuevamente a las tipologías para el diseño, es un conocimiento de tipo empírico, aunque esté sistematizado en estudios que lo hacen asequible para su aplicación.

El texto presentado constituye un resultado que puede aplicarse en la disciplina para facilitar el dominio del tema por parte de los docentes. Su desarrollo permitió plantear algunas preocupaciones o problemas que son de interés para el trabajo metodológico, estas son a modo de propuestas preliminares:

Las relaciones entre metodología de diseño y las técnicas, procedimientos o herramientas que maneja el estudiante en los proyectos, tales como las TIC, las técnicas de investigación social, el dibujo a mano alzada en etapas del proceso creativo entre otros. El papel de la metodología de diseño en la integración con el resto de las disciplinas. La relación entre metodología del diseño y métodos de enseñanza. La relación entre metodología del diseño y organización de la ejecución del proceso docente. La metodología del diseño y el diseño de los temas de proyecto de la carrera.

Resulta estratégico adquirir un modo de enfrentarproceso de diseño conuna fundamentación metodológica sólida. Los problemas específicos son los que pueden variar en cada circunstancia pero la lógica que permite seguir el proceso de trabajo para cualquier caso es algo estratégico. $\mathbf{C}$

\section{Bibliografía.}

Alonso, A. Leyva, C.J, y Campos E. (2012). "Evaluación de impacto ambiental: herramienta en la formación ambiental del arquitecto. Arquitectura y Urbanismo vol. XXXIII, No 3, 2012, ISSN 1815-5898. pp. 38-51. Disponible en: http://scielo.sld.cu/pdf/au/v33n3/au050312.pdf.

Briceño, Á., Gómez, L. J. (2011). "Proceso de Diseño Urbano - Arquitectónico. Provincia [en línea], (enero-junio). (30 de junio de 2018). Disponible en: $<$ http://www.redalyc. org/articulo.oa? $\mathrm{id}=55519834006>\quad$ ISSN 1317-9535.
Cárdenas, E. (1984): "Historia y práctica arquitectónica". Arquitectura y urbanismo, 2, 1984, ISPJAE, La Habana, Cuba.

Fundora, G. (1982): "Información, análisis, síntesis, evaluación: un modelo para la creación en la arquitectura". Revista Arquitectura y Urbanismo, 1. ISPJAE, La Habana.

Fundora, G. (1986): "La proyección arquitectónica docente: Núcleo de la enseñanza". Arquitectura y Urbanismo, No.3. ISPJAE, La Habana, Cuba.

Fundora, G. (1988): "Una significación muy propia: la creación en el estudiante de arquitectura". Revista Cubana de Educación Superior, No 1. La Habana, Cuba.

Gelabert, D., y González, D. Couret. (2013). "Progresividad y flexibilidad en la vivienda. Enfoques teóricos". Arquitecturay Urbanismo, vol. XXXIV, no 1, enero-abril, 2013, p. 17-31, ISSN 1815-5898. Disponible en: http://scielo. sld.cu/pdf/au/v34n1/au030113.pdf.

González, D (2012). "Vivienda, teoría y práctica. Treinta años de experiencia académica en La Habana". Arquitectura y Urbanismo vol. XXXIII, No. 1, 2012, ISSN 1815-5898. Pp 91-104. Disponible en: http://scielo.sld.cu/ pdf/au/v33n1/au070112.pdf.

González, D., Couret, L., Rueda A., González, N., Milián, E. y Llovet, M. (2015). "Evaluación cualitativa de la influencia del diseño arquitectónico en el ambiente interior". Arquitectura y Urbanismo vol. XXXVI, no 3, 2015, ISSN 1815-5898. Pp 54-66. Disponible en: file:///C:/Users/Fernando/Downloads/457858-1-SM\%20(1).pdf.

Granados, M. (2017). "Arquitectura, arte y papel". Contexto. Vol. XI. No15. Septiembre 2017. Pp 27-35. Disponible en: file:///C:/ Users/Fernando/Downloads/DialnetArquitecturaArteYPapel-6336529.pdf.

Hepler, D. y Wallach, P. (1991): "Architecture, Drafting and Design. USA”. GlencoeMcGraw Hill.

Hernández, O. (1985): "Vivienda: tipologías y formas de planeamiento urbano". Arquitectura y urbanismo, No.2, 1985, ISPJAE, La Habana, Cuba.

Miranda, V. y Esteves, M. (2018). "La Arquitectura como disciplina clave para el estudio del Hábitat Rural en climas áridos y (semi) desérticos. El caso de Mendoza, Argentina". Contexto. Vol. XII. Nº16. Marzo 2018. Pp 109125. Disponible en: http://contexto.uanl.mx/ 
index.php/contexto/article/viewFile/147/110.

Moya, L. (1991): "Consideraciones para una teoría de la estética". España: Universidad de Navarra.

Pérez, E. (2001). "El método de diseño en la Disciplina de Proyecto". [Folleto inédito]. Documento docente-metodológico de la Disciplina de Proyecto. Universidad de Camagüey.

Pérez, E. (2004). "Estrategias para la educación en valores en disciplinas de la carrera de Arquitectura". I Encuentro Internacional de Educación en Valores. ISBN 959-16-0252-0. Universidad Ignacio Agramonte y Loynaz, Camagüey.

Pérez, E. (2004). "Lo socio-humanístico, componente esencial en la formación de la identidad del arquitecto". XII Convención Científica de Ingeniería y Arquitectura de la CUJAE. ISBN 959-261-169-6. Pp 4-9.

Prieto, O. (1984): Estudio tipológico de la vivienda camagüeyana en la colonia. Arquitectura y urbanismo, No.2, 1984, ISPJAE. La Habana, Cuba.

Redondo, E., Fonseca, D., Giménez, L., Santana, G., Navarro, I. (2012). "Alfabetización digital para la enseñanza de la arquitectura. Un estudio de caso". Arquitectura Revista [en línea]. 8 (Sin mes). ( 30 de junio de 2018). Disponible en: $<$ http://www.redalyc.org/articulo. oa? id=193623828009> ISSN 1808-5741.

Rodríguez, M. L y Louremy, R. (2007) “El modelo holístico para el proceso de enseñanzaaprendizaje de geometría en arquitectos de la escuela cubana. Revista Latinoamericana de Investigación en Matemática Educativa 10(3): 421-461. Disponible en: http://www. scielo.org.mx/pdf/relime/v10n3/v10n3a6.pdf.

Rozas, E. (1976). “Apuntes sobre metodología del diseño arquitectónico". Santo Domingo: Editorial Taller

Timmling, H. (2009). "Reflexiones en torno al proceso de diseño en arquitectura. Revista AUS [en linea]. (30 de junio de 2018). Disponible en: $<$ http://www.redalyc.org/articulo. oa?id=281722844002 $>$ ISSN 0718-204X.

Trebilcock, M. (2009).'Proceso de Diseño Integrado: nuevos paradigmas en arquitectura sustentable". Arquitetura Revista [en linea], 5 (Julio-diciembre). (30 de junio de 2018). Disponible en: $<$ http://www.redalyc.org/articulo. oa? $\mathrm{id}=193614470001>$ ISSN 1808-5741. 